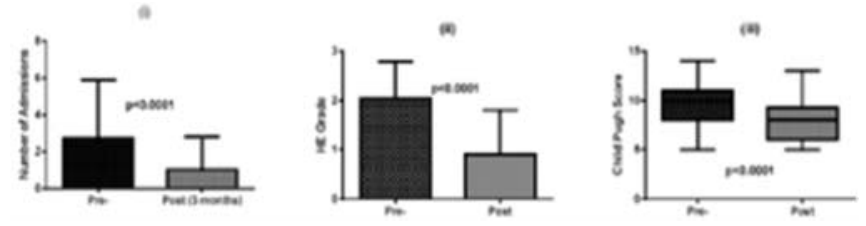

Abstract OC-029 Figure 1

0.0001) [Figure i]. HE grade improved significantly following therapy $(\mathrm{p}<0.0001)$ [Figure ii].

Child Pugh score fell significantly following therapy $(\mathrm{p}<$ 0.0001) [Figure iii], as did MELD and UKELD scores: $15 \pm 7$ vs. $13 \pm 5(\mathrm{p}<0.03)$ and $55 \pm 6$ vs. $51 \pm 5(\mathrm{p}<0.02)$, respectively. This is noteworthy as the MELD score does not include HE as a parameter and is based on bilirubin, INR and creatinine.

Conclusion Our UK multi-centre experience is that rifaximin is well-tolerated and an efficacious treatment for the secondary prevention of HE. Rifaximin significantly reduced both hospital re-admission rates after 3 months treatment, impacting significantly on the NHS resource burden of HE, and reduced overall liver disease severity raising the possibility that its therapeutic effect may extend beyond reducing gut ammonia production.

Disclosure of Interest V. Patel Grant/research support from: Norgine (UK) Ltd., J. Orr Grant/research support from: Norgine (UK) Ltd., J. Sturgeon: None Declared, Z. Habtemariam: None Declared, H. Preedy: None Declared, P. Richardson Consultant for: Norgine (UK) Ltd., R. Aspinall Consultant for: Norgine (UK) Ltd., M. Hudson Consultant for: Norgine (UK) Ltd., D. Shawcross Grant/research support from: Norgine (UK) Ltd., Consultant for: Norgine (UK) Ltd.

\section{OC-030 EFFECTIVE STRATIFICATION OF HEPATOCELLULAR CARCINOMA RISK IN PRIMARY BILIARY CIRRHOSIS: RESULTS OF A MULTI-CENTRE INTERNATIONAL STUDY}

${ }^{1} \mathrm{PJ}$ Trivedi ${ }^{*},{ }^{2} \mathrm{~W}$ Lammers, ${ }^{2} \mathrm{H}$ van Buuren, ${ }^{3} \mathrm{H}$ Janssen, ${ }^{4} \mathrm{P}$ Invernizzi, ${ }^{5} \mathrm{PM}$ Battezzati, ${ }^{6} \mathrm{~A}$ Floreani, ${ }^{7} \mathrm{~A}$ Pares, ${ }^{8} \mathrm{C}$ Ponsioen, ${ }^{9} \mathrm{C}$ Corpechot, ${ }^{10} \mathrm{R}$ Poupon, ${ }^{11} \mathrm{M}$ Mayo, ${ }^{12} \mathrm{~J}$ Talwalkar, ${ }^{13} \mathrm{~A}$ Burroughs, ${ }^{14} \mathrm{~F}$ Nevens, ${ }^{15} \mathrm{~A}$ Mason, ${ }^{16} \mathrm{~T}$ Bruns, ${ }^{1} \mathrm{~K}-\mathrm{K} \mathrm{Li},{ }^{17} \mathrm{~K}$ Kowdley, ${ }^{18} \mathrm{~T}$ Kumagi, ${ }^{18} \mathrm{~A}$ Cheung, ${ }^{19} \mathrm{~A}$ Lleo, ${ }^{20} \mathrm{~N}$ Cazagon, ${ }^{21}$ I Franceschet, ${ }^{22} \mathrm{~L}$ Caballería, ${ }^{23} \mathrm{~K}$ Boonstra, ${ }^{24} \mathrm{E}$ de Vries, ${ }^{12} \mathrm{M}$ Imam, ${ }^{13} \mathrm{G}$ Pieri, ${ }^{25} \mathrm{P}$ Kanwar, ${ }^{12,26} \mathrm{~K}$ Lindor, ${ }^{2} \mathrm{~B}$ Hansen, ${ }^{1} \mathrm{G}$ Hirschfield on behalf of Global PBC Study Group. 'NIHR Biomedical Research Unit and Centre for Liver Research, University of Birmingham, Birmingham, UK; ${ }^{2}$ Department of Gastroenterology and Hepatology, Erasmus Medical Centre, Rotterdam, The Netherlands; ${ }^{3}$ Liver Clinic, Toronto Western and General Hospital, University Health Network, Toronto, Canada; ${ }^{4}$ Liver Unit and Center for Autoimmune Liver Diseases, Humanitas Clinical and Research Center, Rozzano, Italy; ${ }^{5}$ Department of Health Sciences, Università Degli Studi Di Milano, Milano, Italy; ${ }^{6}$ Department of Surgical, Oncological and Gastroenterological, University of Padua, Padua, Italy; ' Liver Unit, Hospital Clínic, CIBERehd, IDIBAPS, University of Barcelona, Barcelona, Spain; ${ }^{8}$ Department of Gastroenterology and Hepatology, Academic Medical Center, Amsterdam, The Netherlands; ${ }^{9}$ Centre de Référence Des Maladies Inflammatoires Des Voies Biliaires, Hôpital Saint-Antoine, APHP, Paris, France; ${ }^{10}$ Centre de Référence Des Maladies Inflammatoires Des Voies Biliaires, Hôpital SaintAntoine, Paris, France; ${ }^{11}$ Digestive and Liver Diseases, UT Southwestern Medical Center, Dallas, USA; ${ }^{12}$ Department Gastroenterology and Hepatology, Mayo Clinic, Rochester, USA; ${ }^{13}$ The Sheila Sherlock Liver Centre, The Royal Free Hospital, London, UK; ${ }^{14}$ Department of Hepatology, University Hospitals Leuven, Leuven, Belgium; ${ }^{15}$ Divison of Gastroenterology and Hepatology, University of Alberta, Edmonton, Canada; ${ }^{16}$ Department of Internal Medicine IV, Jena University Hospital, Friedrich Schiller University Jena, Jena, Germany; ${ }^{17}$ Liver Center of Excellence, Digestive Disease Institute, Virginia Mason Medical Center, Seattle, USA; ${ }^{18}$ Liver Clinic, Toronto Western and General Hospital, Toronto, Canada; ${ }^{19}$ Liver Unit and Center for Autoimmune Liver Diseases, Humanitas Clinical and Research Center, Rozzano, Italy; ${ }^{20}$ Dipartimento Di Scienze Chirurgiche Oncologiche E Gastroenterologiche, Università Degli Studi Di Milano, Milano, Italy; ${ }^{21}$ Department of Surgical, Oncological and Gastroenterological, University of Padua, Padua, Padua, Italy; ${ }^{22}$ Primary Healthcare Centre Premià de Mar, Catalan
Health Institute, Premià de Mar, Spain; ${ }^{23}$ Department of Gastroenterology and Hepatology, Academic Medical Center, Amsterdam, Netherlands; ${ }^{24}$ Department of Gastroenterology and Hepatology, Academic Medical Center, Amsterdam, Netherlands; ${ }^{25}$ Liver Center of Excellence, Digestive Disease Institute, Seattle, USA; ${ }^{26}$ Arizona State University, Phoenix, USA

\subsection{6/gutjnl-2014-307263.30}

Introduction Hepatocellular carcinoma (HCC) is an important but infrequent outcome in primary biliary cirrhosis (PBC). Improved risk evaluation is an important goal for stratified surveillance.

Methods Risk-factor analysis of the 'Global PBC Study Group' comprising 15 centres across North America and Europe spanning >40-years follow-up was performed using Cox proportional hazards model, logistic regression and Kaplan-Meier estimates (SPSSv21).

Results Of 3546 patients with PBC (med. follow-up 8.6 yrs; IQR 4.4-14.1), 131 developed HCC. Excluding those who developed HCC within 12 months of PBC diagnosis ( $\mathrm{n}=$ 23), median time to HCC was 12.7 yrs (6.9-16.8) and subsequent survival 1.1 yrs. (0.2-2.7). At diagnosis, factors associated with HCC development were male gender (adj. HR: 4.4;1.4-12.2, $\mathrm{p}=0.014$ ) and thrombocytopenia (adj. HR: $4.5 ; 1.4-14.8, \mathrm{p}=0.012$ ). Use of ursodeoxycholic acid per-se was not associated with future risk of HCC, but stratification of risk by biochemical response at 12 months was effective by Rotterdam (adj. HR: 8.9;2.1-37.3, P = 0.003), Paris-I (adj. HR: 7.6;2.0-29.0, $\mathrm{p}=0.003$ ) or Toronto criteria (HR: $5.6 ; 1.6-18.8, \mathrm{P}=0.006)$. Five $(4.6$ vs. $0.2 \%)$ and 10 year (13\%vs.1.9\%) HCC incidence was significantly increased for biochemical non-responders $\left(\mathrm{p}=2.2 \times 10^{-9}\right)$, and by multivariate analysis non-response remained the only significant risk factor.

Conclusion Our uniquely powered cohort allows robust demonstration that 12-month biochemical non-response is associated with an increased risk of developing HCC in PBC. Routine surveillance in those achieving biochemical response is unlikely cost-effective.

Disclosure of Interest None Declared.

\section{OC-031 RELAXIN MODULATES CIRRHOSIS-INDUCED RENAL VASCULAR ENDOTHELIAL DYSFUNCTION}

${ }^{1}$ VK Snowdon*, ${ }^{2} \mathrm{PWF}$ Hadoke, ${ }^{3} \mathrm{~W}$ Mungall, ${ }^{2} \mathrm{~A}$ Thomson, ${ }^{1} \mathrm{~T}$ Kendall, ${ }^{2} \mathrm{D}$ Webb, ${ }^{1} \mathrm{JP}$ Iredale, ${ }^{1} \mathrm{JA}$ Fallowfield. ${ }^{1} \mathrm{MRC/Centre}$ for Inflammation Research, University of Edinburgh, Edinburgh, UK: ${ }^{2}$ BHF/University of Edinburgh Centre for Cardiovascular Science, Edinburgh, UK; ${ }^{3}$ Biomedical Research Resources, University of Edinburgh, Edinburgh, UK

\subsection{6/gutjnl-2014-307263.31}

Introduction Hepatorenal syndrome (HRS) is a feared complication of cirrhosis characterised by intense renal vasoconstriction. The pathophysiology remains unclear, pharmacotherapy is limited and mortality is high. We investigated vascular responsiveness and the pathogenesis of renal vasoconstriction in models of advanced rat cirrhosis. Additionally,we determined the mechanism of action of the vasoactive peptide relaxin (RLN), previously shown to increase renal blood flow (RBF) in experimental cirrhosis (Snowdon V et al., BSG 2013).

Methods We induced cirrhosis,reduced RBF and renal dysfunction in male SD rats by carbon tetrachloride (16 wk) or bile duct ligation (4 wk). Arteries from renal (renal, segmental, interlobar) and splanchnic circulation were isolated for functional assessment using wire myography. qPCR array for vasoactive signalling genes, 
western blot for eNOS signalling proteins and NOS activity assay were undertaken in cirrhotic and control kidneys. Markers of oxidative stress and inflammatory cytokines were measured in serum by ELISA. We studied the effects of s.c. infusion of recombinant human RLN(seralaxin; $72 \mathrm{~h}, 4 \mu \mathrm{g} / \mathrm{h}$ ) on these parameters. Doppler USS measured changes in cardiac output (CO) and renal arterial resistive index (RRI) in response to i.v. RLN $(4 \mu \mathrm{g})$. Kidney endothelial morphology was assessed by electron microscopy, $\mathrm{H}+\mathrm{E}$ and PAS stained kidney by light microscopy.

Results In renal arteries from control and cirrhotic rats endothelial vasodilatation was eNOS-dependent. In cirrhotic rats endothelium-dependent relaxation (acetylcholine; 10-9-3 $\times 10$ $5 \mathrm{M})$ was dramatically reduced $(\mathrm{p}<0.0001)$ in all renal arteries, with only a modest reduction seen in the mesenteric arteries. Endothelium-independent relaxation (sodium nitroprusside; 10-9-3 × 10-5 M) and vasoconstriction (phenylephrine; 10-9-3 × 10-5 M) were unaltered. In cirrhotic kidneys, total eNOS expression was up-regulated,as were arginase 2 and caveolin1 (negative regulators of eNOS), and NOS activity was reduced ( $\mathrm{p}<0.05$ ). Acute RLN had no effect on CO but decreased RRI $(p<0.05)$. Extended RLN restored endotheliumdependent relaxation, increased kidney NOS activity $(\mathrm{p}<0.05)$, increased phosphorylated Akt and eNOS, and reduced serum TNF $\alpha$ levels.

Conclusion Renal vascular endothelial dysfunction characterises experimental cirrhosis, through a reduction in renal eNOS activity. This impairment may contribute to the renal vasoconstriction seen in cirrhosis and is a promising target for therapeutic modulation. RLN treatment restored renal endothelial vasodilatation. The potential for recombinant forms of RLN as a haemodynamic modulator in human cirrhosis and HRS merits investigation in translational studies.

Disclosure of Interest None Declared.

\section{OC-032 SURVEILLANCE LEADS TO IMPROVED OUTCOMES FOR PATIENTS WITH HEPATOCELLULAR CARCINOMA (HCC)}

${ }^{1}$ TJS Cross*, ${ }^{1}$ W Ding, ${ }^{1} P D$ Richardson, ${ }^{1} F$ Yousef, ${ }^{2} D$ Palmer, ${ }^{3} E$ Joekes, ${ }^{3} \mathrm{C}$ Farrell, ${ }^{3}$ J Evans. ${ }^{1}$ Department of Gastroenterology and Hepatology, The Royal Liverpool Hospital, Liverpool, UK; ${ }^{2}$ Academic Department of Oncology, The University of Liverpool, Liverpool, UK; ${ }^{3}$ Department of Radiology, The Royal Liverpool Hospital, Liverpool, UK

\subsection{6/gutjn-2014-307263.32}

Introduction 6-monthly ultrasound surveillance is recommended in cirrhotic patients at risk of HCC. The benefit of surveillance has never been demonstrated in a western population.

Methods A retrospective, single centre cohort analysis in patients diagnosed with HCC from 2008-2013. From 2008 an automated recall system for 6-monthly ultrasound was instigated by the radiology department, in preference ad hoc ultrasound requests. Patients with abnormal lesions proceeded to CT, MRI or liver biopsy according to defined international criteria. The primary end-points evaluated were stage of cancer detection (early i.e. BCLC 0 or A), versus late presentation (BCLC B-D) and patient survival from time of diagnosis to 12 months and 60 months.

Results 160 patients were identified. Surveillance status was known in 132 patients. Median patient age was 68 years (57-75), median number of lesions was one, diameter of largest lesion $30 \mathrm{~mm}$ (19-50), and AFP 19.5 (5-250). Patients under surveillance were more likely to have disease at a curative stage 67 vs. $39 \%(\mathrm{p}=0.006$, OR $0.59(0.41-$ 0.84 ), and had better survival at 1 year 80 vs. $62 \%$ ( $\mathrm{p}=$
0.04 , OR $0.77(0.62-0.97)$, and at 5 years 60 vs. $41 \%(\mathrm{p}=$ 0.046 , OR 0.69 (0.48-0.98). On univariate analysis the following variables on survival were evaluated: Age $(\mathrm{p}=0.11)$, Number HCC nodules $(\mathrm{p}=0.31)$, Total diameter of lesions $(\mathrm{p}=0.001)$, Diameter of largest lesion $(\mathrm{p}<0.001)$, AFP $(\mathrm{p}$ $<0.001)$. The presence on imaging of extra-hepatic metastases $(p=0.006)$, lymph nodes $(p=0.004)$, and portal vein thrombosis $\mathrm{p}<0.001)$, were associated with poorer survival.

Conclusion Surveillance for hepatocellular carcinoma leads to earlier diagnosis and improved survival.

Disclosure of Interest None Declared.

\section{OC-033 THE TWEAK AND FN14 PATHWAY AS POTENTIAL MEDIATOR OF LIVER FIBROSIS}

${ }^{1} \mathrm{~A}$ Wilhelm*, ${ }^{1} \mathrm{M}$ Munir, ${ }^{1} \mathrm{E}$ Humphreys, ${ }^{1} \mathrm{D}$ Adams, ${ }^{2} \mathrm{~L}$ Burkly, ${ }^{1} \mathrm{~S}$ Afford, ${ }^{1} \mathrm{C}$ Weston. ${ }^{1}$ Centre for Liver Research, University of Birmingham, Birmingham, UK; ${ }^{2}$ Biogen Idec, Cambridge, USA

\subsection{6/gutjnl-2014-307263.33}

Introduction The TNF superfamily ligand TWEAK and its cognate receptor Fn14 have been implicated in the pathogenesis of liver disease and have been predominantly associated with liver progenitor cell proliferation and ductular reaction. We hypothesised that TWEAK and Fn14 may also be involved in the establishment and progression of fibrosis via a direct effect on hepatic stellate cell (HSC) function.

Methods TWEAK and Fn14 expression was studied by qPCR, western blot and immunostaining in tissue and stromal cells from explanted human liver specimens and normal donor livers surplus to surgical requirements, or as a byproduct of surgical resection. The responses of HSCs to TWEAK were investigated by western blot, live cell imaging and proliferation assays. TWEAK was measured in HSC supernatant by ELISA.

Results Confocal microscopy revealed localisation of Fn14 to cells expressing stromal markers in normal human livers, with significant upregulation in diseased livers. Fn14 expression was confirmed in both primary human HSCs and myofibroblasts in vitro. Stimulation with recombinant TWEAK led to an upregulation of NF-kB signalling and induced proliferation in cultured HSCs. TWEAK immunostaining localised the protein to the fibrotic areas of ALD and NASH liver sections suggestive of an autocrine regulation of Fn14 signalling. We confirmed that HSCs express TWEAK and release it into their environment by qPCR and ELISA, and demonstrated that function-blocking TWEAK antibodies reduced the proliferative capacity of HSC.

Conclusion Our study suggests that TWEAK/Fn14 promotes liver fibrosis via enhanced proliferation of HSC, possibly through an autocrine mechanism driven by HSC production of TWEAK.

Disclosure of Interest None Declared.

\section{OC-034 OUTCOME OF PATIENTS CONSIDERED UNSUITABLE FOR LIVER TRANSPLANTATION - A MISSED OPPORTUNITY FOR PALLIATIVE CARE?}

A Phoolchund*, S Murray, B Hogan, J O'Beirne. Royal Free Hospital and UCL Institute of Liver and Digestive Health, Royal Free Hospital, London, UK

10.1136/gutjnl-2014-307263.34 